\title{
Efecto crónico del Body Jump sobre medidas de condición física y salud: Un estudio piloto Chronic effect of Body Jump on physical conditioning and health measures: A pilot study
}

Andrea Amorós Illán, Alejandro López-Valenciano, FranciscoAyala Rodríguez, Irene Wesley López, Iñaki Ruiz-Pérez, María del Pilar García-Vaquero, Sergio Hernández-Sánchez

Universidad Miguel Hernández de Elche(España)

Resumen. El BodyJump ${ }^{\circledR}$ es una disciplina que ha irrumpido fuertemente en los últimos años en los centros deportivos. Pese a ello, no hay evidencias que permitan respaldar su uso como programa eficaz y seguro para la mejora de la condición física y salud. Por lo tanto, el objetivo principal de este estudio fue analizar el efecto de un programa de corta duración de BodyJump ${ }^{\circledR}$ sobre la condición física y salud. Un total de tres mujeres adultas sedentarias (edad: 23 años; 55,3 \pm 2,8 kg; 161,3 \pm 7,1 cm) completaron este estudio de casos siendo sometidas a un programa de BodyJump ${ }^{\circledR}$ durante cuatro semanas. Un total de 17 medidas de condición física y salud y dos medidas relacionadas con sintomatología del tendón de Aquiles y rotuliano fueron definidas como variables dependientes. El efecto del programa de BodyJump ${ }^{\circledR}$ sobre las variables dependientes fue determinado a través del estadístico tamaño del efecto. Un tamaño del efecto pequeño ( $d=0,5-1,25)$ fue encontrado en las medidas relacionadas con la fuerza y resistencia de la musculatura del tronco, potencia de salto vertical con contra-movimiento y equilibrio dinámico. Para el resto de medidas de condición física y salud se observó un cambio trivial o no relevante tras la intervención $(\mathrm{d}<0,5)$. Los hallazgos del presente estudios informan de que un programa de BodyJump ${ }^{\circledR}$ podría ser considerado una actividad eficiente para la mejora de la fuerza y resistencia muscular del tronco, potencia y equilibrio dinámico en mujeres adultas jóvenes sedentarias. Palabras clave: fuerza muscular, salud, fitness, ejercicio físico, resistencia cardiorrespiratoria

Abstract. BodyJump ${ }^{\circledR}$ is a discipline that has broken out strongly in recent years in fitness centers. However, there is no evidence to support its use as an effective and safe program for improving fitness and health. Therefore, the main objective of this study was to analyze the effect of a short-term BodyJump ${ }^{\circledR}$ program on fitness and health. A total of three sedentary adult women (age: 23 years, $55.3 \pm 2.8 \mathrm{~kg}, 161.3 \pm 7.1 \mathrm{~cm}$ ) completed this case study undergoing a BodyJump ${ }^{\circledR}$ program for four weeks. A total of 17 measures of fitness and health and two measures related to symptoms of Achilles and patellar tendon were defined as dependent variables. The effect of the BodyJump ${ }^{\circledR}$ program on the dependent variables was determined through the statistical effect size. A small effect size $(\mathrm{d}=0.5-1.25)$ was found in measures related to trunk musculature strength and endurance, power of countermovement vertical jump and dynamic balance. For the rest of physical and health measures, a trivial or non-relevant change was observed after the intervention $(\mathrm{d}<0.5)$. The findings of the current study report that a short BodyJump ${ }^{\circledR}$ program could be considered an efficient activity for improving trunk muscle strength, endurance, and dynamic balance in sedentary young-adult women.

Key words: muscle strength, health, fitness, physical activity, aerobic capacity.

\section{Introducción}

En los últimos años, la industria del fítness ha experimentado un crecimiento vertiginoso en los países desarrollados (Boned, Felipe, Barranco, Grimaldi-Puyana \& Crovetto, 2015). Así, cada vez son más las alternativas de actividades y programas de ejercicio físico que son ofertadas por los centros deportivos con el propósito de mejorar la salud de sus practicantes (Gallegos, López, Valeiras \& Suárez, 2011; Yan \& McCullagh, 2004).

Dentro de esta amalgama de posibilidades, las actividades físicas de carácter colectivo y dirigidas por un instructor certificado se han convertido en una de las opciones prioritarias entre la población adulta (GarcíaFerrando \& Llopis, 2011; Schiffer, Kleinert, Sperlich, Schulte \& Strüder, 2009). La alta popularidad de este tipo de actividades podría radicar en el uso de una base músical rock-pop actualizada junto a una constante retroalimentación positiva que parece favorecer un clima motivacional agradable para los practicantes; pero sobre todo por la promesa de una mejora general en la condición física y salud con tan sólo unos minutos de ejercicio físico (30-60 minutos) realizados 2-3 días a la semana.

En este sentido, una de las actividades que ha irrumpido con más fuerza en muchos centros deportivos es el BodyJump ${ }^{\circledR}$, conocido también bajo el nombre de JumpFit, PowerJump o Jump Training. El BodyJump ${ }^{\circledR}$ ha sido definido como «un programa de entrenamiento que promueve la mejora de la condición física general a través de coreografías ejecutadas sobre una superficie elástica con variación de ritmo y movimientos, de forma interválica y con bajo impacto» (Furtado, Simão \& Lemos, 2004, p. 371). Cada sesión de BodyJump ${ }^{\circledR}$ presenta una duración de 55 minutos aproximadamente, en donde se llevan a cabo coreografías formadas por la combinación de movimientos sencillos.

Sin embargo, y a diferencia de otras actividades físicas dirigidas como el BodyPump ${ }^{\circledR}$ (Greco et al., 2011; Rustaden, Haakstad, Paulsen

Fecha recepción: 20-02-17. Fecha de aceptación: 16-07-17 Alejandro Lopez Valenciano alejandro.lopezv@umh.es
\& Bø, 2017) y Spinning ${ }^{\circledR}$ (Bianco et al., 2010; Valle, Mello, Fortes, Dantas \& Mattos, 2010; Verrusio et al., 2016), el ascenso meteórico de la popularidad del BodyJump ${ }^{\circledR}$ entre la población adulta (sobre todo mujeres) no se ha visto acompañado de una suficiente evidencia científica que permita respaldar los supuestos beneficios que se le atribuyen. En este sentido, no se han encontrado (desde el conocimiento de los autores) estudios científicos que examinen el efecto crónico del BodyJump $^{\circledR}$ sobre variables de condición física y salud. Por lo tanto, son necesarios estudios científicos que analicen el efecto crónico del BodyJump ${ }^{\circledR}$ sobre medidas de condición física y salud antes de promover su uso en la población como actividad segura y eficiente para la mejora de la misma.

Así, el objetivo principal del presente estudio fue analizar los efectos de cuatro semanas de BodyJump ${ }^{\circledR}$ sobre medidas de condición física y salud en mujeres adultas jóvenes.

\section{Método}

\section{Diseño del estudio}

Este estudio de casos presentó un diseño con estructura clásica de sesión pre-test (valoración inicial), programa de intervención $\left(\right.$ BodyJump $^{\circledR}$ ) y sesión post-test (valoración final).

Como variable independiente se estableció el programa de intervención: BodyJump ${ }^{\circledR}$. Como variables dependientes se eligieron un total de 17 medidas de condición física y salud agrupadas en siete bloques (composición corporal, fuerza máxima, resistencia muscular, potencia de la extremidad inferior, flexibilidad, agilidad y fitness cardiorrespiratorio). Finalmente, y dado el volumen de saltos ejecutados por los participantes en cada una de las sesiones, se valoró la posible aparición de sintomatología de tendinopatías en el tendón de Aquiles y rotuliano tras la fase de intervención a través de los cuestionarios VISAA (Robinson, et al., 2001) y VISA-P (Hernández-Sánchez, Hidalgo \& Gómez, 2011) respectivamente.

Antes de la fase de intervención, el valor inicial de cada participante para cada una de las variables dependientes fue determinado a través de dos sesiones de evaluación, separadas entre sí 72 horas. Cada sesión de 
evaluación a la que cada una de las participantes fue sometida tuvo una duración total de aproximadamente 45 minutos. Una vez completadas las dos sesiones de evaluación inicial, las participantes fueron integradas en el programa de BodyJump ${ }^{\circledR}(n=4)$. Así, una semana después de la última sesión pre-test, se inició la fase de intervención, que tuvo una duración de cuatro semanas y una frecuencia semanal de 3 días (12 sesiones).

La fase de intervención tuvo una duración de 4 semanas por haber demostrado ser suficiente para conseguir adaptaciones metabólicas (Gibala \& McGee, 2008) y músculo esqueléticas (Gibala, et al., 2006) clínicamente relevantes tras programas de ejercicio físico supervisado con parámetros de la carga similares al BodyJump ${ }^{\circledR}$ (frecuencia semanal, intensidad, volumen) en población adulta.

La evaluación final de la eficacia del programa de intervención $\left(\right.$ BodyJump $^{\circledR}$ ) sobre las diferentes variables objeto de estudio, cuyo contenido y procedimiento fue idéntico al de la evaluación inicial, tuvo lugar a las 72 horas después de completar la última sesión de la fase de intervención. Tanto las sesiones de evaluación como la fase de intervención fueron llevadas a cabo por dos estudiantes de último curso del grado en Ciencias de la Actividad Física y del Deporte (CAFD) bajo la tutela de un doctor en Ciencias del Deporte.

\section{Participantes}

Un total de cuatro mujeres adultas jóvenes participaron en el presente estudio. Las participantes fueron seleccionadas de forma no probabilística y categorizadas como sedentarias de acuerdo a la puntuación obtenida (<450 METs) en el «Cuestionario Internacional de Actividad Física (IPAQ)» en su formato reducido (Booth, Ainsworth, Pratt, Ekelund, Yngve, Sallis \& Oja, 2003). Como criterios de exclusión del presente estudio se establecieron: a) presentar algún signo y síntoma de enfermedad cardiovascular, metabólica y musculo-esquelética que pudiese verse agravado por la realización de las diferentes pruebas de evaluación propuestas y/o por la realización del programa de intervención que es objeto de estudio (determinado a través del cuestionario PAR-Q+); b) realizar otro tipo de actividad física complementaria que no sea la propuesta por el presente estudio durante el periodo de intervención; c) haber participado en un programa de entrenamiento de BodyJump ${ }^{\circledR}$ en el mes previo al estudio; d) no cumplir con la frecuencia de entrenamiento de tres días a la semana durante una de las cuatro semanas que dura la fase de la intervención; e) abandonar el programa de intervención antes de su completa finalización (cuatro semanas); y f)no asistir a una o varias de las sesiones de evaluación.

Antes de ser evaluados, todas las participantes fueron informadas verbalmente y por escrito de los posibles riesgos y procedimientos del presente estudio, y posteriormente todas firmaron un consentimiento informado aprobado por el comité de ética de una universidad. Finalmente tres mujeres jóvenes (edad: 23 años; masa corporal: 55,3 $\pm 2,8 \mathrm{~kg}$; estatura: 161,3 \pm 7,1 cm) completaron el presente estudio, siendo una participante eliminada del mismo por incumplir el criterio de inclusión

\section{Procedimiento}

\section{Medidas de condición física y salud}

Previo a la realización de las diferentes pruebas de evaluación de la condición física y salud, cada participante completó un calentamiento dinámico general, el cual consistió en 6-8 minutos de ejercicios de locomoción a una intensidad auto-percibida de «ligera» (60-65\% frecuencia cardiaca máxima), seguido de estiramientos dinámicos de los principales grupos musculares (isquiosurales, cuádriceps, aductores y tríceps sural).

Posteriormente se evaluaron 17 medidas de condición física y salud agrupadas en siete bloques:

1) Composición corporal: se estimaron los valores de masa corporal (kg), porcentaje de masa grasa y porcentaje de masa muscular a través del empleo de una báscula especializada (TANITA UM-081).

2) Fuerza máxima: se determinó de forma indirecta el valor (fórmula de Brzycky [1993]) en kg de la repetición máxima de los ejercicios de press banca horizontal (extremidad superior) y sentadilla (extremi- dad inferior) siguiendo la metodología descrita por Naclerio, Jiménez, Alvar \& Peterson (2009).

3) Resistencia muscular: se valoró la resistencia muscular de los principales núcleos articulares de la extremidad superior y tronco a través de la batería de pruebas propuesta por la American College of Sports Medicine (2013): a) press banca YMCA; b) push up test; y c) curl up test. Asimismo, se implementaron varias pruebas más para la valoración de la fuerza resistencia de los músculos del tronco: a) side bridge test (McGill, Childs \& Liebenson, 1999); b) Ito test modificado (Juan-Recio, Barbado, López-Valenciano \& Vera-García, 2014); y c) Sorensen test (Biering-sørensen, 1984).

4) Flexibilidad: se valoró la flexibilidad de la musculatura posterior del muslo (V sit and reach test) siguiendo el protocolo establecido por Sainz de Baranda, Ayala, Cejudo \& Santonja (2012).

5) Fitness cardiorrespiratorio: se determinó el valor del $\mathrm{VO}_{2}$ máx a partir de la prueba de Astrand y Ryhming (American College of Sports Medicine, 2013).

6) Potencia tren inferior: se empleó la batería de tests propuesta por Meylan, Mcmaster, Cronin, Mohammad, Rogers \& Deklerk (2009), que consta de tres acciones de salto diferentes; a) salto con contra movimiento (CMJ) vertical con pierna dominante; b) $\mathrm{CMJ}$ horizontal con pierna dominante; y c) CMJ lateral con pierna dominante.

7) Evaluación del equilibrio dinámico: la prueba $Y$ Balance ${ }^{\circledR}$ fue utilizada para determinar el equilibrio dinámico global de la extremidad inferior (Gribble, Hertel \& Plisky, 2012).

Las pruebas anteriormente citadas y empleadas para la obtención de los parámetros relacionados con la condición física y salud fueron seleccionadas entre una amplia batería de test, recurriendo a su bajo nivel de exigencia física y a su utilización en diversos estudios donde se evaluaba la condición física en participantes con similares características a los del presente estudio (Greco et al., 2012; Smith, Snow, Fargo, Buchanan \& Dalleck, 2016). Se estableció un orden predeterminado para su ejecución a fin de evitar o minimizar las posibles interacciones negativas que se pudiesen producir entre las diferentes pruebas de evaluación (tabla 1). Tres intentos fueron efectuados para cada prueba (con excepción de las pruebas de fuerza muscular máxima y fitness cardiorrespiratorio, donde se efectuaron dos intentos), seleccionando la media de todos los intentos para el posterior análisis estadístico.

Tabla 1

Estructura del proceso de evaluación de las medidas de condición física y salud antes y después de la aplicación del programa de intervención

1 Sintomatología lesión tendinosa

2 Composición corporal

3 Fuerza muscular máxima

4 Sesión de evaluación 2

5 Equilibrio dinámico

6. Resistencia muscular

7. Flexibilidad

8. Fitness cardiovascular

\section{BodyJump ${ }^{\circledR}$}

En todas las sesiones de entrenamiento se llevó a cabo la misma estructura y contenidos de BodyJump ${ }^{\circledR}$. En este sentido, cada una de las 12 sesiones de entrenamiento llevadas a cabo estuvo dividida en tres fases (tabla 2), las cuales estaban amenizadas por música, empezando con un calentamiento (8-12 minutos); seguido de una parte principal (30-35 minutos) donde se combinan distintos ejercicios al ritmo de la música sobre el mini trampolín; para finalmente acabar la sesión con una fase de vuelta a la calma (8-12 minutos)

La fase de calentamiento consistió en una coreografía sencilla que englobaba movimientos básicos de locomoción y movilidad articular de la extremidad superior e inferior, y posteriormente una pequeña secuencia sobre el mini trampolín de aprendizaje de los movimientos básicos a realizar en la parte principal. La intensidad fue creciendo gradualmente a lo largo de esta fase de calentamiento hasta alcanzar un carácter del esfuerzo auto-percibido de «ligero - algo duro» (escala de Borg).

La parte principal de la sesión constaba de ejercicios realizados sobre el mini trampolín durante siete canciones, alcanzando dos picos de intensidad máxima de manera progresiva mediante coreografías de 
Tabla 2

\begin{tabular}{|c|c|c|c|c|c|}
\hline Parte de la sesión & Canción & Duración (s) & $\begin{array}{c}\text { Intensidad } \\
\text { (escala de Borg) }\end{array}$ & & Ejemplos de ejercicios \\
\hline \multirow{5}{*}{ Calentamiento } & 1 & 236 & Ligera & Estiramientos dinámicos (10 rep) & $\begin{array}{cc}\text { Ejercicios en trampolín: } \\
\text { Abducción de cadera unilateral }\end{array}$ \\
\hline & \multirow{4}{*}{2} & \multirow{4}{*}{251} & \multirow{4}{*}{ Ligera-Moderada } & Isquiosurales & Abducción de cadera unilateral \\
\hline & & & & Psoas & Talones al glúteo \\
\hline & & & & Cuádriceps & Saltos unilaterales y bilaterales \\
\hline & & & & & Skipping bajo \\
\hline \multirow{6}{*}{ Parte Principal } & 3 & 240 & Moderada & \multicolumn{2}{|c|}{ Ejercicios en trampolín alternando movimiento de brazos y pierna } \\
\hline & 4 & 225 & Moderada-Duro & Saltos laterales (unilateral y bilateral) & Abrir y cerrar piernas con cruce de brazos \\
\hline & 5 & 251 & & $\begin{array}{l}\text { Skiltos laterales (uniletal y Dilateral) } \\
\text { Skipping bajo }\end{array}$ & Talones al glúteo \\
\hline & 6 & 255 & Ligera & Skipping alto & Jogging \\
\hline & 7 & 228 & Moderada & Sentadillas & Saltos unilaterales \\
\hline & $\begin{array}{l}8 \\
9\end{array}$ & $\begin{array}{l}237 \\
280\end{array}$ & $\begin{array}{l}\text { Moderada } \\
\text { Duro }\end{array}$ & Salto en $\mathrm{V}$ & Giros de cadera \\
\hline \multirow{5}{*}{ Vuelta a la calma } & \multirow[b]{2}{*}{10} & \multirow[b]{2}{*}{247} & \multirow[t]{2}{*}{ Dris } & \multicolumn{2}{|c|}{ Fortalecimiento del tronco (2-3 series de $10-15$ rep) } \\
\hline & & & & Encorvamientos & Bicicleta \\
\hline & \multirow{3}{*}{11} & \multirow{3}{*}{270} & & \multicolumn{2}{|c|}{ Estiramientos estáticos (2-3 series de 30s) } \\
\hline & & & - & Isquiosurales & Pectoral \\
\hline & & & & $\begin{array}{l}\text { Cuadriceps } \\
\text { Psoas }\end{array}$ & Deltoides \\
\hline
\end{tabular}

S: segundos; rep: repeticiones

menor intensidad separadas por una canción de recuperación. Asimismo, el segundo pico de intensidad fue seguido por una canción de vuelta a la calma. En los picos de máxima intensidad se alcanzaba una intensidad subjetiva (escala de Borg) de «algo duro-duro» (estimación del 5589\% frecuencia cardiaca máxima [FCmáx]).

La última parte de la sesión de entrenamiento o vuelta a la calma estaba compuesta por una pequeña coreografía sin el mini trampolín donde se primaba el trabajo de fuerza y resistencia de la musculatura del tronco, 2-3 series de 10-15 repeticiones de 2-3 ejercicios (cinco minutos), para posteriormente finalizar con 2 series de 30 segundos de 3-5 ejercicios de estiramientos estáticos de los principales grupos musculares (isquiosurales, cuádriceps, psoas, dorsal, pectoral, tríceps) (cinco minutos).

\section{Análisis estadístico}

Los estadísticos media y diferencias pre y post intervención fueron empleados para describir las variables.

Al tratarse de un estudio de tres casos, el empleo de pruebas estadísticas no paramétricas de comparación de medidas relacionadas podría no ser apropiado dada su fuerte vinculación con el tamaño muestral. Así, los cambios en los valores de cada variable dependiente ocasionados post-intervención fueron analizados a través del empleo del estadístico tamaño del efecto (Cohen, 1988), cuya vinculación al tamaño muestral es reducida y de acuerdo a la siguiente fórmula:

$$
\begin{gathered}
d=c(n-1) \frac{\text { Ypre }- \text { Ypost }}{\text { Spre }} \\
c(n-1)=1-\frac{3}{4 n-5}
\end{gathered}
$$

Donde Ypre e Ypost es el valor medio de cada variable en el pre-test y post-test respectivamente, Spre es la desviación estándar de cada variable en el pre-test y n es el tamaño muestral.

En particular, y para la interpretación del tamaño del efecto se empleó la escala propuesta por Rhea (2004) para el entrenamiento de fuerza en personas no entrenadas: $<.5=$ trivial; .5-1.25 = pequeño; $>1.25$ - 2 = moderado; $>2$ =grande.

\section{Resultados}

En la tabla 3 se muestran los resultados obtenidos por las tres participantes en las sesiones de evaluación pre y post intervención para las medidas de condición física y salud. El tamaño del efecto del BodyJump ${ }^{\circledR}$ fue pequeño ( $\left.\mathrm{d}=.5-1.25\right)$ para las variables relacionadas con la resistencia de la musculatura del tronco estimadas a través de las pruebas curl up test, side bridge e Ito test; así como para la potencia y equilibrio dinámico de la extremidad inferior medidas a través de la altura de salto vertical y la distancia anterior alcanzada en la prueba Y Balance

Tabla 3

Estadísticos descriptivos de los resultados obtenidos en la evaluación pre-test y post-test para las medidas de condición física relacionada con la salud separadas según participantes. Las diferencias en valores absolutos entre sesiones de evaluación para cada una de los constructos han sido igualmente expuestas. El tamaño del efecto ha sido también computado para los tres

\begin{tabular}{|c|c|c|c|c|c|c|c|c|c|c|}
\hline $\begin{array}{c}\text { Prueba } \\
\text { Variable } \\
\end{array}$ & \multicolumn{3}{|c|}{ Participante 1} & \multicolumn{3}{|c|}{ Participante 2} & \multicolumn{3}{|c|}{ Participante 3} & $\begin{array}{c}\text { Tamaño del } \\
\text { efecto }\end{array}$ \\
\hline Masa corporal (kg) & 58.3 & 58.72 & 0.4 & 55.6 & 55.8 & 0.2 & 52.3 & 52.6 & 0.3 & 0.07 \\
\hline $\operatorname{IMC}\left(\mathrm{Kg} / \mathrm{m}^{2}\right)$ & 20.7 & 20.8 & 0.1 & 21.2 & 21.3 & 0.1 & 22.1 & 22.2 & 0.1 & 0.10 \\
\hline Masa Magra (kg) & 39.6 & 40.5 & 0.9 & 40.4 & 41.1 & 0.7 & 365 & 37.2 & 0.7 & 0.27 \\
\hline \multicolumn{11}{|c|}{ Fitness cardiovascular } \\
\hline $\mathrm{Vo}_{2} \max \left(\mathrm{kg} \times \mathrm{m} \times \min ^{-1}\right)$ & 29.1 & 32.4 & 3.3 & 50.4 & 51.9 & 1.5 & 42.1 & 47.5 & 5.4 & 0.23 \\
\hline \multicolumn{11}{|c|}{ Fuerza muscular maxima $(\mathrm{Kg})$} \\
\hline Tren superior & 38.2 & 40.6 & 2.4 & 27.2 & 30 & 2.8 & 32.7 & 38.2 & 5.5 & 0.47 \\
\hline Press Banca YMCA (rep) & 5 & 7 & 2 & 7 & 11 & 4 & 16 & 17 & 1 & 0.29 \\
\hline Push up test (rep) & 7 & 8 & 1 & 15 & 13 & -2 & 33 & 24 & -9 & -0.18 \\
\hline Curl Up test (rep) & 3 & 9 & 6 & 36 & 70 & 34 & 9 & 14 & 5 & 0.62 \\
\hline Biering-Sorensen (s) & 102 & 107 & 5 & 113 & 115 & 2 & 73 & 87 & 14 & 0.25 \\
\hline Side Bridge (s) & 49 & 67 & 18 & 36 & 63 & 27 & 54 & 55 & 1 & 1.2 \\
\hline Ito test $(\mathrm{s})$ & 44 & 62 & 18 & 45 & 68 & 27 & 70 & 84 & 14 & 0.91 \\
\hline \multicolumn{11}{|c|}{ Flexibilidad $(\mathrm{cm})$} \\
\hline V sit and reach & 6 & 6 & 0 & -6 & -5 & 1 & 3 & 1 & -2 & 0.04 \\
\hline Anterior & 61.8 & 63.7 & 1.9 & 64.5 & 67 & 2.5 & 59.3 & 61.3 & $2 /$ & 0.6 \\
\hline Posterior & 52.4 & 55.7 & 3.3 & 73.5 & 76 & 2.8 & 64 & 71.3 & 6.5 & 0.3 \\
\hline Postero Lateral & 44.8 & 46 & 1.2 & 75 & 76 & 1 & 62 & 68 & 6 & 0.13 \\
\hline \multicolumn{11}{|c|}{ Sintomatología de lesión tendinosa } \\
\hline VISA-A & 100 & 100 & 0 & 100 & 100 & 0 & 100 & 100 & 0 & 0 \\
\hline VISA-P & 100 & 100 & 0 & 100 & 100 & 0 & 100 & 100 & 0 & 0 \\
\hline
\end{tabular}
participantes entores absol

kg: kilogramos; IMC: índice de masa corporal; rep: repeticiones; cm: centímetros; s: segundos; CMJ: salto con contra-movimiento; m: metro; min: minuto 
respectivamente. Para el resto de medidas de condición física y salud se observó un cambio trivial o no relevante tras la intervención $(\mathrm{d}<.5)$.

\section{Discusión}

Los principales hallazgos del presente estudio de casos informan, por un lado, de que un programa de BodyJump ${ }^{\circledR}$ de corta duración (cuatro semanas) realizado tres veces a la semana (12 sesiones) podría ser un estímulo suficiente para provocar efectos positivos $(\mathrm{d}>$.5) sobre variables de resistencia muscular de los flexores, extensores e inclinadores del tronco en mujeres adultas jóvenes sedentarias. Esta mejora en la resistencia de la musculatura del tronco podría estar explicada por las altas demandas a las que dicha musculatura se ve sometida con el fin de mantener una correcta estabilidad central durante los distintos ejercicios ejecutados sobre una superficie inestable como es el trampolín; pero sobre todo, a la ejecución de un volumen alto de trabajo específico de la misma (2-3 series de 10-15 repeticiones de 2-3 ejercicios) en la última parte de todas las sesiones de BodyJump ${ }^{\circledR}$. Estas mejoras obtenidas en la resistencia de la musculatura flexora, extensora e inclinadora del tronco tras el programa de BodyJump ${ }^{\circledR}$ de corta duración podrían tener implicaciones importantes para la prevención y tratamiento del dolor lumbar. En este sentido, estudios previos han demostrado que una reducida fuerza y resistencia de la musculatura del tronco es uno de los principales factores de riesgo de dolor lumbar (Taylor, Goode, George \& Cook, 2014), una de las patologías más prevalentes (Walker, 2000)e incapacitantes en el mundo (Hoy, et al., 2014).

Por otro lado, los resultados de este estudio también informan de que el programa de BodyJump ${ }^{\circledR}$ produjo ligeras mejoras en la potencia muscular (altura de salto vertical) $(\mathrm{d}=1.03)$ y equilibrio dinámico (distancia anterior en la prueba $\mathrm{Y}$ Balance $\left.{ }^{\circledR}\right)(\mathrm{d}=.6$ ) de las participantes. Es posible que la realización de ejercicios de salto en trampolín durante las sesiones de BodyJump ${ }^{\circledR}$ haya producido un incremento en la rigidez de la unidad músculo-tendón, lo cual puede haber impactado positivamente en el retraso electromecánico (mecanismo de feedback del sistema sensoriomotor), asociado éste a procesos de acoplamiento excitación-contracción. Así, este posible cambio en la rigidez de la unidad músculo-tendón ha podido, por un lado, mejorar el ciclo de estiramiento-acortamiento, permitiendo una mejora en su capacidad para absorber y transmitir energía elástica durante acciones de salto con contramovimiento (Maffiuletti, Aagaard, Blazevich, Folland, Tillin \& Duchateau, 2016); y por otro lado, mejorar la coordinación neuromuscular, lo que permite un mejor equilibrio y estabilidad dinámica (Anderson \& Behm, 2005). Estas mejoras en potencia muscular y equilibrio podrían igualmente suponer, entre otros aspectos, un menor riesgo de caídas (Ambrose, Paul \& Hausdorff, 2013) y una mayor eficiencia durante la ejecución de movimientos (Fort-Vanmeerhaeghe \& Romero-Rodríguez, 2013).

Del mismo modo, es importante resaltar que aunque el programa de BodyJump ${ }^{\circledR}$ no produjo un efecto estadísticamente relevante, si parece existir una fuerte tendencia indicando mejoras en las variables de fuerza máxima, tanto de la extremidad superior $(\mathrm{d}=.47)$ como de la inferior (d $=.49$ ). Quizás esta posible tendencia positiva en las variables anteriormente citadas pudiese indicar la necesidad de mayor tiempo (semanas) de aplicación del programa de intervención para reportar resultados relevantes. Por el contrario, el programa de BodyJump ${ }^{\circledR}$ no produjo mejoras relevantes en las variables relacionadas con la composición corporal (\% de grasa corporal, IMC y masa grasa) y fitness cardiovascular ( $\mathrm{VO}_{2}$ máx). Estudios previos informan de que la intensidad promedio alcanzada durante una sesión de BodyJump ${ }^{\circledR}$ en adultos oscila entre el 81-87\% de la FCmáx (Furtado et al., 2004; Grossl, Guglielmo \& Carminatti, 2008; Perantoni, Deresz, Lauria, Lima \& Novaes, 2009). Este intervalo de intensidad se encuentra dentro de la categoría de moderada-vigorosa, la cuál ha sido propuesta como la óptima para mejorar la composición corporal y fitness cardiovascular por instituciones tan prestigiosas como la American College of Sports Medicine (Garber, et al., 2011; Thompson, Gordon \& Pescatello, 2010) y la National Strength and Conditioning Association (Clayton, Drake, Larkin, Linkul, Martino,
Nutting \& Tumminello, 2015). Por tanto, y a diferencia de las variables relacionadas con la fuerza y resistencia muscular, es posible que la mejora en la composición corporal y fitness cardiovascular requiera, al igual que las variables de fuerza máxima, de programas de entrenamiento con mayor duración (semanas) y/o volumen de trabajo (días por semana). Esta hipótesis se sustenta en el hecho de que otra actividad colectiva con similares valores de intensidad promedio como es el Spinning ${ }^{\circledR}$ (Battista, Foster,Andrew, Wright, Lucia \& Porcari, 2008; Caria, Tangianu, Concu, Crisafulli \& Mameli, 2007; López-Miñarro \& Muyor, 2010) ha demostrado ser una intervención eficaz para la mejora de la composición corporal tras ser aplicadas durante al menos 12 semanas (Bianco et al., 2010; Valle et al., 2010; Verrusio et al., 2016).

Finalmente, los resultados del presente estudio parecen asimismo indicar que un programa de BodyJump ${ }^{\circledR}$ de corta duración no produjo sintomatología de lesión tendinosa. Sin embargo, y dado la corta duración del programa (cuatro semanas), estos resultados deben de ser tomados con cautela, ya que este tipo de patologías suelen estar asociados a procesos de sobrecarga de mayor duración (semanas) (Dixit, Difiori, Burton \& Mines, 2007).

Dado que el actual estudio es el primero (desde el conocimiento de los autores) que ha analizado el efecto de un programa de BodyJump ${ }^{\circledR}$ sobre variables de condición física y salud, las comparaciones con estudios previos han sido imposibles.

Aunque el presente estudio es novedoso en ciertos aspectos (número de pruebas exploratorias, diseño, estructura del programa de intervención), ciertas limitaciones propias de los estudios de casos deben ser resaltadas. Así, las principales limitaciones son: a) el pequeño tamaño muestral utilizado $(\mathrm{n}=3)$ que impide realizar generalizaciones, aunque si una aproximación al objeto de estudio; y b) la corta duración del programa. Por tanto, son necesarios más estudios que analicen el efecto de programas de BodyJump ${ }^{\circledR}$ de larga duración (> 4 semanas) sobre variables de condición física y salud a través de diseños longitudinales de medidas repetidas con grupo control (no intervención), grupo placebo y grupo experimental en amplias muestras para aclarar el verdadero efecto del BodyJump ${ }^{\circledR}$ como actividad segura y eficiente para la mejora de la salud

\section{Conclusiones}

Los resultados del presente estudio de casos sugieren que la práctica regular del BodyJump ${ }^{\circledR}$ podría ser una actividad eficiente para la mejora de la fuerza máxima y resistencia muscular del tronco, potencia y equilibrio dinámico en adultos sedentarios. Por lo tanto, atendiendo a estos hallazgos relacionados con la mejora de la condición física y salud y al probado impacto positivo que sobre la adherencia y motivación presenta la práctica de ejercicio físico en grupo, supervisada por un instructor certificado y con base musical rock-pop; el BodyJump ${ }^{\circledR}$ podría ser una herramienta apropiada para luchar contra las altas tasas de abandono que durante los primeros meses presentan personas que se inician en la práctica de actividad física para la salud en los centros fitness. .

\section{Financiación}

Este trabajo no ha sido financiado.

\section{Conflicto de intereses}

Los autores declaran no tener ningún conflicto de intereses.

\section{Referencias}

Ambrose, A.F., Paul, G., \& Hausdorff, J. M. (2013). Risk factors for falls among older adults: a review of the literature. Maturitas, 75(1), 51-61.

American College of Sports Medicine (2013). ACSM's guidelines for exercise testing and prescription. Philadelphia, PA; Lippincott Williams y Wilkins.

Anderson, K., \& Behm, D. G. (2005). The impact of instability resistance 
training on balance and stability. Sports Medicine, 35(1), 43-53.

Battista, R.A., Foster, C., Andrew, J., Wright, G., Lucia, A., \& Porcari, J. P. (2008). Physiologic responses during indoor cycling. Journal of Strength \& Conditioning Research, 22(4), 1236-1241.

Bianco, A., Bellafiore, M., Battaglia, G., Paoli, A., Caramazza, G., Farina, F., \& Palma, A. (2010). The effects of indoor cycling training in sedentary overweight women. Journal of Sports Medicine and Physical Fitness, 50(2), 159-165.

Biering-Sørensen, F. (1984). Physical measurements as risk indicators for low-back trouble over a one-year period. Spine, 9(2), 106-119.

Boned, C.J., Felipe, J.L., Barranco, D., Grimaldi-Puyana, M., \& Crovetto, M. (2015). Professional profile of workers in spanish fitness clubs. Revista Internacional de Medicina y Ciencias de la Actividad Fiìsica y el Deporte, 15(58), 195-210

Booth, M.L., Ainsworth, B.E., Pratt, M., Ekelund, U., Yngve, A., Sallis, J.F., \& Oja, P. (2003). International physical activity questionnaire: 12-country reliability and validity. Medicine and Science in Sports and Exercise, 195, 3508-1381.

Brzycki, M. (1993). Strength testing-predicting a one-rep max from reps-to-fatigue. Journal of Physical Education, Recreation \& Dance, 64 (1), 88-90.

Caria, M.A., Tangianu, F., Concu, A., Crisafulli, A., \& Mameli, O. (2007). Quantification of Spinning ${ }^{\circledR}$ bike performance during a standard 50minute class. Journal of Sports Sciences, 25(4), 421-429.

Clayton, N., Drake, J., Larkin, S., Linkul, R., Martino, M., Nutting, M., \& Tumminello, N. (2015). Foundations of Fitness Programming. Colorado: National Strength and Conditioning Association.

Cohen, J. (1988). Statistical power analysis for the behavioral sciences (2nd ed.). Hillsdale, NJ: Lawrence Earlbaum Associate

Dixit, S., Difiori, J.P., Burton, M., \& Mines, B. (2007). Management of patellofemoral pain syndrome. American Family Physician, 75(2), 194-202.

Fort Vanmeerhaeghe, A., \& Romero Rodriguez, D. (2013). Rol del sistema sensoriomotor en la estabilidad articular durante las actividades deportivas. Apunts Medicina de lo Esport, 48(178), 69-76.

Furtado, E., Simão, R., \& Lemos, A. (2004). Análise do consumo de oxigênio, freqüência cardíaca e dispêndio energético, durante as aulas do JumpFit. Revista Brasileira de Medicina do Esporte, 10(5), 371375

García-Ferrando, M., \& Llopis, R. (2011). Ideal democrático y bienestar personal. Encuesta sobre los hábitos deportivos en España 2010. Madrid: Consejo Superior de Deportes, Centro de Investigaciones Sociológicas.

Gallegos, A.G., López, M.G., Valeiras, J.A.A., \& Suárez, N.R. (2011). Motivos de práctica en el ámbito de la actividad física no competitiva. Espiral. Cuadernos del Profesorado, 4(7), 15-22.

Garber, C.E., Blissmer, B., Deschenes, M.R., Franklin, B.A., Lamonte, M.J., I-Min Lee, M.D., Nieman, D.C., \& Swain, D.P. (2011). Quantity and quality of exercise for developing and maintaining cardiorespiratory, musculoskeletal and neuromotor fitness in apparently healthy adults: guidance for prescribing exercise. Medicine and Science in Sports and Exercise, 43(7), 1334-1359.

Gibala, M.J., \& McGee, S.L. (2008). Metabolic adaptations to short-term high-intensity interval training: a little pain for a lot of gain?. Exercise and Sport Sciences Reviews, 36(2), 58-63.

Gibala, M.J., Little, J.P., Van Essen, M., Wilkin, G.P., Burgomaster, K.A. Safdar, A., ... \& Tarnopolsky, M.A. (2006). Short term sprint interval versus traditional endurance training: similar initial adaptations in human skeletal muscle and exercise performance. Journal of Physiology, 575(3), 901-911.

Greco, C.C., Oliveira, A.S., Pereira, M.P., Figueira, T.R., Ruas, V.D., Goncalves, M., \& Denadai, B. S. (2011). Improvements in metabolic and neuromuscular fitness after 12-week Bodypump ${ }^{\circledR}$ training. Journal of Strength and Conditioning Research, 25(12), 3422-3431.

Gribble, P.A., Hertel, J., \& Plisky, P. (2012). Using the Star Excursion Balance Test to assess dynamic postural-control deficits and outcomes in lower extremity injury: a literature and systematic review. Journal of Athletic Training, 47(3), 339-357.

Grossl, T., Guglielmo, L.G.A., \& Carminatti, L.J. (2008). Determinação da intensidade da aula de powerjump por meio da freqüência cardíaca. Revista Brasileira de Cineantropometria y Desempenho Humano, 10(2), 129-136.

Hernandez-Sanchez, S., Hidalgo, M.D., \& Gomez, A. (2011). Crosscultural adaptation of VISA-P score for patellar tendinopathy in
Spanish population. Journal of Orthopaedicy Sports Physical Therapy, 41(8), 581-591.

Hoy, D., March, L., Woolf, A., Blyth, F., Brooks, P., Smith, E., ... \& Burstein, R. (2014). The global burden of neck pain. Annals of the Rheumatic Diseases, (7), 1309-1315.

Juan-Recio, C., Barbado, D., López-Valenciano, A., \& Vera-García, F.J. (2014). Test de campo para valorar la resistencia de los músculos del tronco. Apunts: Educación Física y Deportes, 117, 59-68.

López-Miñarro, P.A., \& Muyol, J. (2010). Heart rate and overall ratings of perceived exertion during Spinning ${ }^{\circledR}$ cycle indoor session in novice adults. Science \& Sports, 25(5), 238-244.

Maffiuletti, N.A., Aagaard, P., Blazevich, A.J., Folland, J., Tillin, N., \& Duchateau, J. (2016). Rate of force development: physiological and methodological considerations. European Journal of Applied Physiology, 116(6), 1091-1116.

McGill, S.M., Childs, A., \& Liebenson, C. (1999). Endurance times for low back stabilization exercises: clinical targets for testing and training from a normal database. Archives of Physical Medicine and Rehabilitation, 80(8), 941-944.

Meylan, C., Mcmaster, T., Cronin, J., Mohammad, N.I., Rogers, C., \& Deklerk, M.(2009) Single-leg lateral, horizontal, and vertical jump assessment: reliability, interrelationships, and ability to predict sprint and change-of-direction performance. Journal of Strength and Conditioning Research, 23(4), 1140-1147.

Naclerio, F.J., Jiménez, A., Alvar, B.A., \& Peterson, M.D. (2009). Assessing strength and power in resistance training. Journal of Human Sport and Exercise, 4(2), 110-113.

Perantoni, C.B., Deresz, C.S., Lauria, A.A., Lima, J.R.P., \& Novaes, J.S. (2009). Análisis de la intensidad de una sesión de Jump Training. Fitness \& Performance Journal, 8(4), 286-290.

Rhea, M. R. (2004). Determining the magnitude of treatment effects in strength training research through the use of the effect size. Journal of Strength and Conditioning Research, 18(4), 918-920.

Robinson, J.M., Cook, J.L., Purdam, C., Visentini, P.J., Ross, J., Maffulli, N., ... \& Khan, K M. (2001). The VISA-A questionnaire: a valid and reliable index of the clinical severity of Achilles tendinopathy. British Journal of Sports Medicine, 35(5), 335-341.

Rustaden, A.M., Haakstad, L. A., Paulsen, G., \& Bø, K. (2017). Effects of BodyPump and resistance training with and without a personal trainer on muscle strength and body composition in overweight and obese women-A randomised controlled trial. Obesity Research \& Clinical Practice, in press, DOI: http://dx.doi.org/10.1016/j.orcp.2017.03.003

Sainz de Baranda, P., Ayala, F., Cejudo, A., \& Santonja, F. (2012). Descripción y análisis de la utilidad de las pruebas sit-and-reach para la estimación de la flexibilidad de la musculatura isquiosural. Revista Española de Educación Física y Deportes, 396, 119-133.

Schiffer, T., Kleinert, J., Sperlich, B., Schulte, S., \& Strüder, H. K. (2009). Effects of aerobic dance and fitness programme on physiological and psychological performance in men and women. I.J. Fitness, 5(2), 37-46.

Smith, L. E., Snow, J., Fargo, J. S., Buchanan, C. A., \& Dalleck, L. C. (2016) The Acute and Chronic Health Benefits of TRX Suspension Training ${ }^{\circledR}$ in Healthy Adults. International Journal of Research in Exercise Physiology, 11(2), 1-15.

Taylor, J.B., Goode, A.P., George, S.Z., \& Cook, C.E. (2014). Incidence and risk factors for first-time incident low back pain: a systematic review and meta-analysis. Spine Journal, 14(10), 2299-2319.

Thompson, W.R., Gordon, N.F. \& Pescatello, L.S. (2010). ACSM's Guidelines for exercise testing and prescription. (8 $8^{\mathrm{a}}$ ed.) Philadelphia: Wolters Kluwer/Lippincott Williams \& Wilkins.

Valle, V.S.D., Mello, D.B.D., Fortes, M., Dantas, E.H.M., \& Mattos, M. A. D. (2010). Effect of diet and indoor cycling on body composition and serum lipid. Arquivos Brasileiros de Cardiologia, 95(2), 173178.

Verrusio, W., Andreozzi, P., Renzi, A., Martinez, A., Longo, G., Musumeci, M., \& Cacciafesta, M. (2016). Efficacy and safety of spinning exercise in middle-aged and older adults with metabolic syndrome: randomized control trial. Annali dell'Istituto Superiore di Sanità, 52(2), 295300.

Walker, B.F. (2000). The prevalence of low back pain: a systematic review of the literature from 1966 to 1998. Clinical Spine Surgery, 13(3), 205-217.

Yan, J.H., \& McCullagh, P. (2004). Cultural influence on youth's motivation of participation in physical activity. Journal of Sport Behavior, 27(4), 378. 